\title{
Optical constants in the ultraviolet region of evaporated high index thin films
}

\author{
K V S R APPARAO and T C BAGCHI \\ Spectroscopy Division, Bhabha Atomic Research Centre, Trombay, Bombay 400085, India
}

\begin{abstract}
A suitable method to determine the optical constants of high index thin films is essential for developing high efficiency dielectric thin film devices in the uv region from $240 \mathrm{~nm}$ to $400 \mathrm{~nm}$. A quick and accurate method is established to determine these constants. Using this method the optical losses, refractive index, absorption coefficient and extinction coefficient of $\mathrm{ZrO}_{2}$ films prepared by the method of reactive evaporation were evaluated in the UV region.
\end{abstract}

Keywords. Thin films; refractive index; optical constants; absorption coefficient; extinction coefficient.

\section{Introduction}

Knowledge of optical losses and constants of thin films is essential for the development of efficient low-loss high reflecting dielectric mirrors for different laser and Fabri-Perot interferometer applications in the Uv region from $240 \mathrm{~nm}$ to $400 \mathrm{~nm}$. Such applications require suitable high index $(n \geq 2 \cdot 0$ ) optical films with minimum extinction coefficient $\left(k \leq 5 \times 10^{-4}\right)$. Very few high index films are known to be suitable for uv region and even for these materials complete data of optical constants and their dependence on the various process parameters used during the preparation of the films are not available. Development work to study and establish such thin film data for each material involves preparing as many as 60 sample films each deposited with different process parameters and determining the optical constants of each sample film accurately. A quick and accurate method is established to determine the optical constants of thin films using the transmittance spectrum of the film.

\section{Experimental}

$\mathrm{ZrO}_{2}$ films were prepared on a $25 \mathrm{~mm}$ diameter fused silica substrates by evaporating optical grade $\mathrm{ZrO}_{2}$ using the electron beam gun in a conventional $500 \mathrm{~mm}$ vacuum coating unit. A total of 35 samples of $\mathrm{ZrO}_{2}$ films were prepared for the present studies each with different evaporation parameters. For all the samples prepared the uniformity of film thickness over the whole surface was found to be within $\pm 2 \%$ of the average thickness. The transmittance spectrum of each sample substrate was recorded using double beam spectrophotometer (Hitachi model 330, photometric accuracy $\pm 0.2 \%$ 


\section{Method of determination}

Optical losses and constants of each sample film were determined using its transmittance spectrum. A typical spectrum of about $300 \mathrm{~nm}$ thick $\mathrm{ZrO}_{2}$ film of refractive index $n$ deposited on a substrate of index $s$ is shown in figure 1 . The transmittance spectrum of the substrate before coating the film is also shown in figure 1 .

\subsection{Optical losses}

The percentage optical losses $L$ of thin films include losses due to bulk absorption $A$, scattering $S$, fluorescence $F$ and are given by

$$
L=(A+S+F)=100-(R+T),
$$

where $R$ and $T$ are the specular reflection and transmission of the film respectively. It is well known that the transmittance spectrum of a dielectric film consists of a series of transmittance maxima and minima corresponding to the interference fringes as shown in figure 1 . For a film of zero losses the peak maxima $T_{\mathrm{PM}}$ (corresponding to even multiples of half wave optical thickness) should be the same as the substrate transmittance $T_{s}$ at respective wavelength points. The attenuation of $T_{\mathrm{PM}}$ as seen in figure 1 at the lower wavelengths can be attributed to the losses in the film. The percentage losses $L$ of the film at wavelength $\lambda_{0}$ were determined from the spectrum using the relation

$$
L=100\left(T_{\mathrm{s}}-T_{\mathrm{PM}}\right)
$$

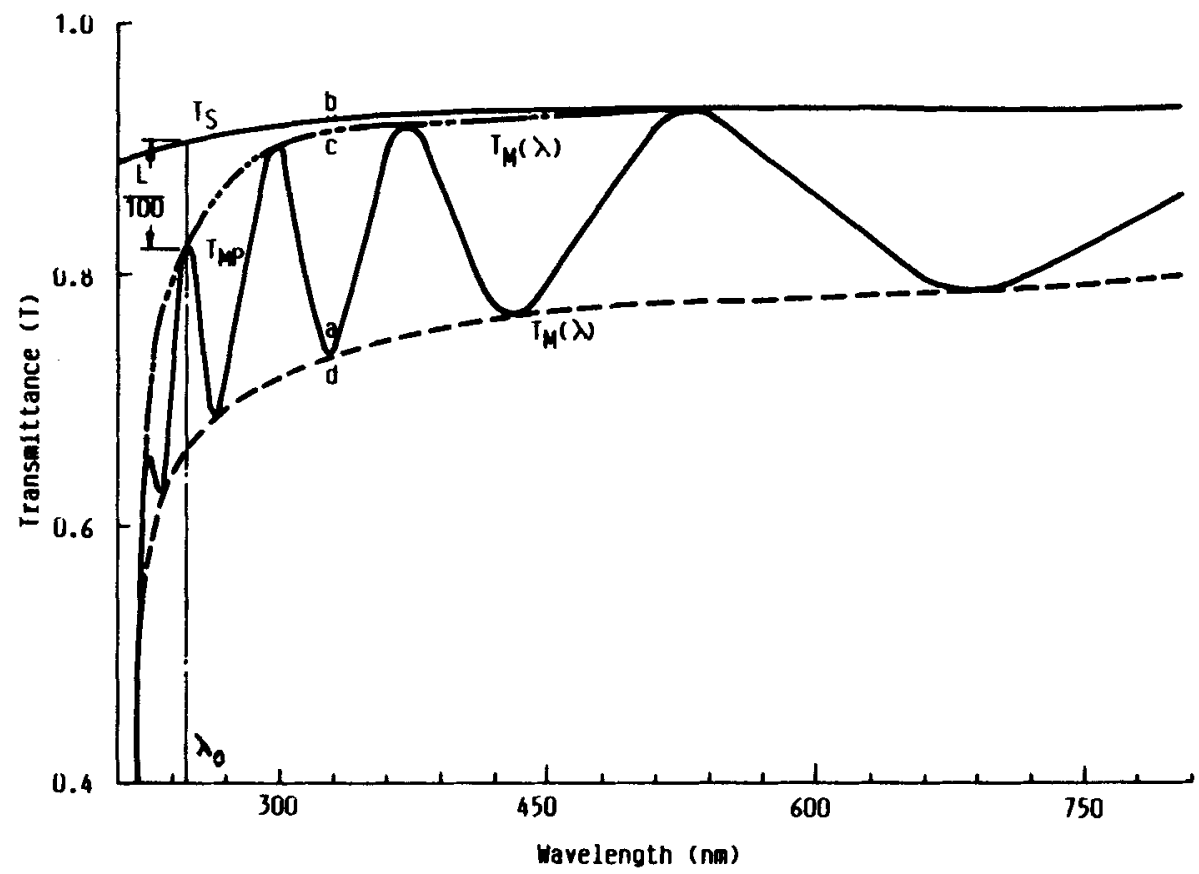

Figure 1. Transmittance spectrum of (a) $300 \mathrm{~nm}$ thick $\mathrm{ZrO}_{2}$ film of index $n$ coated on a substrate index of $s$, (b) the substrate before coating, (c) continuous function $T_{M}(\lambda)$ and (d) the continuous function $T_{m}(\lambda)$. 

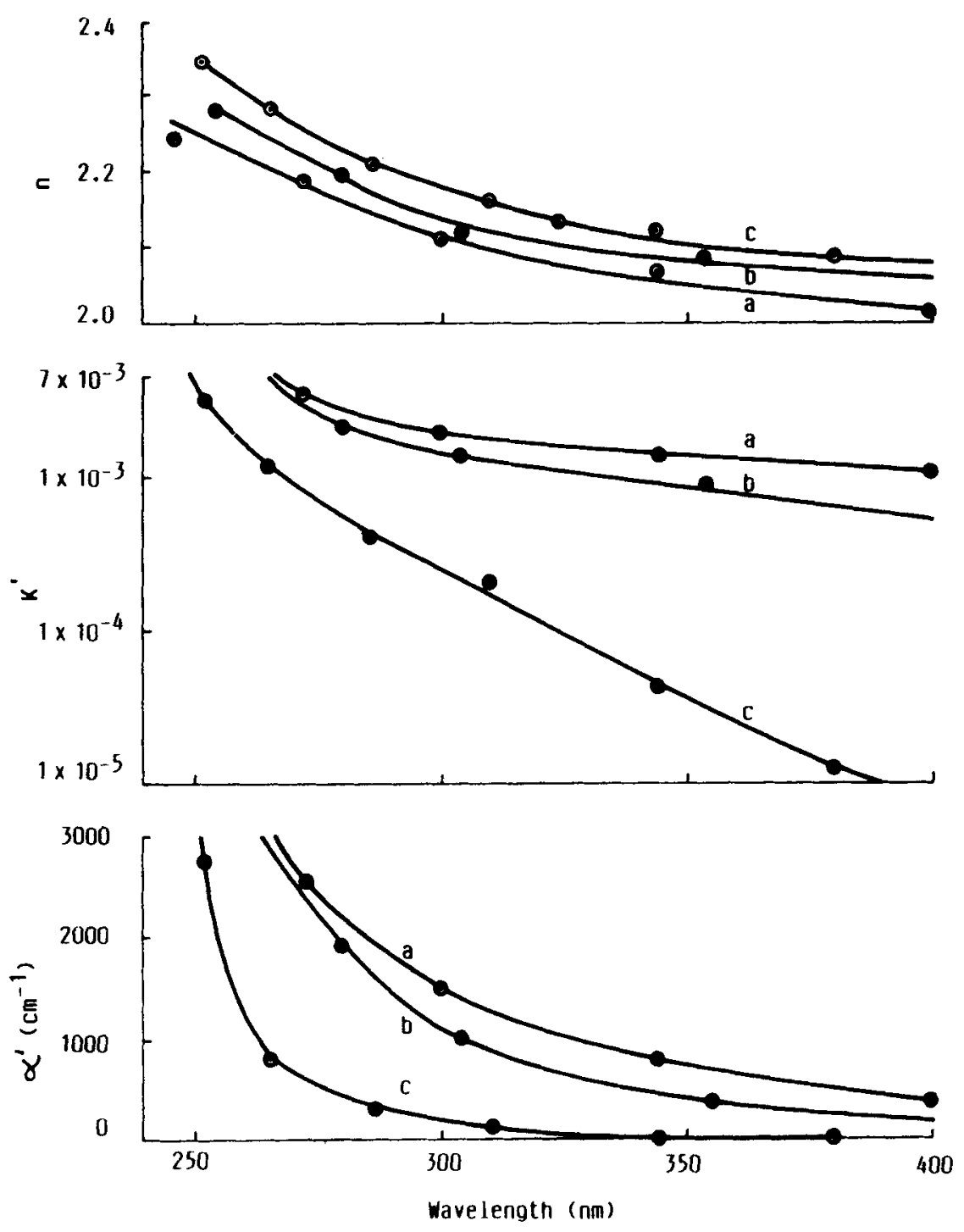

Figure 2. Dependence of optical constants of $\mathrm{ZrO}_{2}$ film on rate of evaporation. (a) $20 \AA / \mathrm{sec}$, (b) $10 \AA / \mathrm{sec}$ and (c) $5 \AA / \mathrm{sec} . t \simeq 275 \mathrm{~nm}, T=260^{\circ} \mathrm{C}$ and $O_{2}=2 \times 10^{-4} \mathrm{mbar}$.

\subsection{Optical constants}

The refractive index $n$, absorption coefficient $\alpha$, extinction coefficient $k$ of the film at different wavelength points and its physical thickness $t$ were determined by the method of Swanepoel (1983) using the continuous functions $T_{M}(\lambda)$ and $T_{m}(\lambda)$ as shown in figure 1. The formulae used in this method to compute $\alpha$ and $k$ were derived assuming that the attenuation of the functions $T_{M}(\lambda)$ and $T_{m}(\lambda)$ at shorter wavelength region was due to pure absorption losses in the film. But in practice thin films prepared by vacuum evaporation suffer losses due to scattering and fluorescence in addition to absorption. So the constants $k$ and $\alpha$ deduced by this method are now called effective constants $k^{\prime}$ and $\alpha^{\prime}$. 
Table 1. Optical losses and constants $(275 \mathrm{~nm}$ thick $) \mathrm{ZrO}_{2}$ films in the uv region.

\begin{tabular}{|c|c|c|c|c|}
\hline \multirow{2}{*}{$\begin{array}{c}\text { Wavelength } \\
\lambda \\
\mathrm{nm}\end{array}$} & \multirow{2}{*}{$\begin{array}{c}\text { Optical losses } \\
L \\
\%\end{array}$} & \multicolumn{3}{|c|}{ Optical constants } \\
\hline & & $n$ & $10^{3} \mathrm{~cm}^{\alpha^{\prime}}$ & $k^{\prime}$ \\
\hline 250 & 8.00 & $2 \cdot 35$ & $3 \cdot 547$ & $7.0 \times 10^{-3}$ \\
\hline 300 & 0.60 & $2 \cdot 16$ & $0 \cdot 192$ & $4.7 \times 10^{-4}$ \\
\hline 350 & 0.13 & $2 \cdot 10$ & 0.031 & $8.5 \times 10^{-5}$ \\
\hline 400 & 0.03 & 2.06 & 0.010 & $5.0 \times 10^{-5}$ \\
\hline
\end{tabular}

\section{Results and discussion}

The measured percentage losses and constants were plotted against wavelength for all the film samples of $\mathrm{ZrO}_{2}$ films each prepared with different evaporation conditions. The results of the relevant samples were grouped into different sets. Each set of films were prepared under identical conditions but with one parameter variable. Figure 2 shows the results of one such set wherein the dependence of optical constants of $\mathrm{ZrO}_{2}$ films on rate of evaporation is shown. The optical losses and refractive index at $250 \mathrm{~nm}$ ( $L=8 \%$ and $n=2.35$ ) for our films of $275 \mathrm{~nm}$ thick were found to be comparable with those films prepared by the method of sputtering $(L=9 \%$ and $n=2.47)$ as reported by Pawlewicz et al (1980). The losses of these films in the visible region above $440 \mathrm{~nm}$ are less than $0.01 \%$ and the constants at $515 \mathrm{~nm}$ are found to be compatible with those reported by Pulker (1976). The optical constants and losses at different wavelength points for the $\mathrm{ZrO}_{2}$ films ( $275 \mathrm{~nm}$ thick) as prepared by us are given in table 1 .

\section{References}

Pawlewicz W T, Hays D D and Martin P M 1980 Thin Solid Films 73169

Pulker H K 1976 Thin Solid Films 34343

Swanepoel J 1983 J. Phys. E16 1214 